\title{
The role of optimization to control traffic signals setting on capacity and flow at peak hours at intersections
}

\author{
Pegah Jafari Haghighatpour ${ }^{1 *}$, Ali Mansourkhaki ${ }^{2}$, Gholamreza Mehdizadeh ${ }^{3}$,Mahmouddreza Keymanesh ${ }^{4}$ \\ ${ }^{1}$ PhD Candidate in Transportation of Tehran Payame Noor University, Tehran \\ ${ }^{2}$ Associate Professor, Department of Civil Engineering, University of Science and Technology, Tehran, Iran \\ ${ }^{3}$ Master of Science, Department of Engineering, Islamic Azad University South Tehran Branch \\ ${ }^{4}$ Assistant Professor, Department of Civil Engineering, Tehran Payame Noor University, Tehran, Iran \\ *Corresponding author E-mail: p.j.haghighatpour@gmail.com
}

Copyright $\odot 2015$ Pegah Jafari Haghighatpour et al. This is an open access article distributed under the Creative Commons Attribution License, which permits unrestricted use, distribution, and reproduction in any medium, provided the original work is properly cited.

\begin{abstract}
Control the pre timing of traffic signals has many advantages because of its responsiveness to traffic demands, short cycles, and effective use of capacity leading to and recovering from oversaturation and amenability to aggressive transit priority. Traffic congestion in urban road and freeway networks leads to a strong degradation of the network infrastructure and accordingly reduced throughput, which can be countered via suitable control measures and strategies. Coordination between intersections in a central system increases capacity, appropriate traffic flow and reduce total delays of vehicles. Scats system has been installed in many world intersections, and many researchers have done about the advantages of it to decrease delays and travel times at intersection. SCATS is a modular system and development of this is possible. Now in Tehran's this system is used and traffic conditions matches on it. Traffic signal timing schedule gives in this system as default and although this system is capable of adapting to the moment traffic but in terms of super saturation and during peak hours due to the lack of optimization, default program around intersection has caused widespread congestion at intersection. In this paper, two intersections of East and West of Tehran have been selected, and in two different situations, the flow to capacity ratio of traffic signal timing has been investigated before and after optimization. Simulation by AIMSUN and optimization by SYNCHRO software is done. After optimization can be observed that if before the pre-defined schedule for SCATS, this plan for each intersection of the volume of traffic at peak hours has been optimized, a great reduction in delay and increase in capacity can be observed at intersections. For example, delay time reduction has been occurred about 14.77 in AM peak time and 12.65 in PM peak time at GOLBARG-DARDASHT intersection.
\end{abstract}

Keywords: Delay; Intersection; Flow and Capacity; Optimization; Traffic Signals Setting.

\section{Introduction}

Traffic congestion is a major problem in modern societies due to increasing population and economic activity. This motivates the need for better utilizing the existing infrastructures and for efficiently controlling the traffic flow in order to minimize the impact of congestion.

Transportation has always been a crucial aspect of human civilization, but it is only in the second half of the last century that the phenomenon of traffic congestion has become predominant due to the rapid increase in the number of vehicles and in the transportation demand in virtually all transportation modes. Traffic congestion appears when too many vehicles attempt to use a common transportation infrastructure with limited capacity.

The emergence of traffic (i.e., many interacting vehicles using a common infrastructure) and subsequently traffic congestion (whereby demand temporarily exceeds the infrastructure capacity) have opened new innovation needs in the transportation area.

One of the key tools for influencing the efficiency of traffic flow is traffic signal control that enables conflicting traffic to flow through intersections via the timing of green/red light cycles. 
It has long been recognized that the challenge is to find optimal cycle timing over many intersections. In contrast, vehicle-actuated controls use online measurements from on-road detectors (e.g., inductive loops) to optimize signal timings on a cycle-to-cycle basis in real time.

Some examples of commonly used implementations are: SCOOT [1], UTOPIA [2], and the hierarchical scheme RHODES. Combinations of both the fixed time plan and vehicle-actuated control also exist; one widely used example is SCATS [3].

Given a choice of the control scheme, various approaches to optimize the Signal. The aim of this paper is to determine the factors affecting the optimal timing of traffic signals at the intersection and Modeling and optimization of an effective step in reducing delays, travel time, balancing flow and capacity of urban intersection.

\section{Literature review}

Aleksandra Stevanovic. Et al in paper title " Assessing the Ageing of Pre-Timed Traffic Signal Control Using Synchro and SimTraffic investigate the ageing of pre-timed traffic control through the use of Synchro and SimTraffic. The first section of the paper presents the concept of ageing of signal timing plans.

The second part estimates the benefits of optimized timing plans in Synchro in the micro simulation environment of SimTraffic. The theoretical nine-node grid network is used as a test bed to model 21 scenarios of deterministic and 100 scenarios of stochastic traffic demand and distribution changes in link flows.

The research does not prove the existence of unique ageing measures, which could correlate any change in link traffic flows with the benefits of signal retiming. The results show that for networks with unchanged traffic distributions, there is a benefit of up to $3 \%$ for up to $5 \%$ of uniform growth in traffic demand. When stochastic variations of traffic demand and distribution are introduced, the benefits rise to an average of $35 \%$ [4].

Byungkyu Park .et al in paper "Evaluating the impacts of urban corridor traffic signal optimization" investigates the impacts of traffic signal timing optimization on vehicular fuel consumption and emissions at an urban corridor.

The traffic signal optimization approach proposed integrates a TRANSIMS microscopic traffic simulator, the VT-Micro model (a microscopic emission and fuel consumption estimation model), and a genetic algorithm (GA)-based optimizer. An urban corridor consisting of four signalized intersections in Charlottesville, VA, USA, is used for a case study. The result of the case study is then compared with the best traffic signal timing plan generated by Synchro using the TRANSIMS microscopic traffic simulator.

The proposed approach achieves much better performance than that of the best Synchro solution in terms of air quality, energy and mobility measures: $20 \%$ less network-wide fuel consumption [5].

Byungkyu "Brian" Park, et al provide a paper " Stochastic Optimization for Sustainable Traffic Signal Control".

The primary research focus of traffic Signal control systems have been the development of traffic signal timing plans minimizing vehicular delay and stops. Very little is known if such a strategy were optimal for a sustainable traffic signal control system minimizing emission and fuel consumption.

This paper presents a development of a sustainable traffic signal control system and speed management framework consisting of a microscopic simulation model.

Based on an implementation of the proposed framework on a case study network, it was found that the proposed framework was very effective in minimizing delay and stops.

\section{Control loop}

The Traffic flow behavior in the (road or freeway or mixed) traffic Network depends on some external quantities that are classified in to two groups [6]:

- Control inputs that are directly related to corresponding Control devices such as traffic Lights, variable message signs, etc.; the control inputs May be selected from an admissible control region Subject to technical, physical, and operational Constraints/

- Disturbances, whose values cannot be manipulated, But may be measurable (e.g., demand) or detectable or predictable over a future time horizon.

\section{Road traffic control}

Traffic lights at intersections are the major control measure in urban road networks.

Traffic lights were originally installed in order to guarantee the safe crossing of antagonistic streams of vehicles and pedestrians; with steadily increasing traffic demands, it was soon realized that, once traffic lights exist, they may lead (under equally safe traffic conditions) to more or less efficient network operations, hence there must exist an optimal control strategy leading to minimization of the total time spent by all vehicles in the network[7] a although the corresponding optimal control problem may be readily formulated for any road network, its real-time solution.

And realization in a control loop. Faces a number of apparently insurmountable difficulties. 
- The red-green switching's of traffic lights call for the introduction of discrete variables, which renders the optimization problem combinatorial.

- $\quad$ The size of the problem for a whole network is very large.

- Many unpredictable and hardly measurable disturbances (incidents, illegal parking, pedestrian crossings, intersection blocking, etc.) may perturb the traffic flow.

- Measurements of traffic conditions are mostly local (via inductive loop detectors) and highly noisy due to various effects.

- $\quad$ There are tight real-time constraints, e.g., decision making within $2 \mathrm{~s}$ for advanced control systems.

- The combination of these difficulties renders the solution of a detailed optimal control problem infeasible for more than one intersection. Therefore, proposed control strategies for traffic control introduce a number of simplifications of different kinds or address only a part of the related traffic control problems.

There are four possibilities for influencing traffic conditions via traffic lights operation:

- Stage specification: For complex intersections involving a large number of streams, the specification of the optimal number and constitution of stages is a nontrivial task that can have a major impact on intersection capacity and efficiency.

- $\quad$ Split: This is the relative green duration of each stage (as a portion of the cycle time) that should be optimized according to the demand of the involved streams.

- Cycle time: Longer cycle times typically increase the intersection capacity because the proportion of the constant lost times becomes accordingly smaller; on the other hand, longer cycle times may increase vehicle delays in under saturated intersections due to longer waiting times during the red phase.

- Offset: This is the phase difference between cycles for successive intersections that may give rise to a "green wave" along an arterial; clearly, the specification of offset should ideally take into account the possible existence of vehicle queues. Control strategies employed for road traffic control may be classified according to the following characteristics.

- $\quad$ Fixed-time strategies for a given time of day (e.g., morning peak hour) are derived off-line by use of appropriate optimization codes based on historical constant demands and turning rates for each stream; traffic-responsive strategies make use of real-time measurements (typically one or two inductive loops per link) to calculate in real time the suitable signal settings.

- Isolated strategies are applicable to single intersections while coordinated strategies consider an urban zone or even a whole network comprising many intersections.

- The first weakness to be addressed is that phases can form an erratic, unpredictable order in the previously proposed Back Pressure policy.

This is acceptable in the context of communications systems but for urban road traffic this is undesirable since erratic ordering of phases brings frustration to drivers and potentially causes confusion leading to dangerous actions.

Move over, if one inbound road is particularly backlogged, then it is possible that other roads are "starved" by being assigned a red light for an extended period.

The second weakness that we address is that prior applications have required each intersection to know the "turning fractions," that is, the fraction of traffic from each inbound road that will turn into each possible outbound road. We prove that the stability results still apply when these turning fractions are estimated using even very simple measurements; specifically, any unbiased estimator of the turning fractions suffices.

To test the practicality of the theoretical refinements described above, we also present the numerical comparison of the proposed Back Pressure algorithm with the approach of L"ammer[8] without switching cost.

The results suggest that our cyclic phase Back Pressure policy tends to outperform other distributed polices both in terms of throughput and congestion [9].

Although the performance of each policy varies widely depending on the parameter setting such as cycle length or decision frequency, under the optimal setting, the Back Pressure with cyclic phase and without cyclic phase have better throughput in compare with the other policies.

\section{Methodology}

Several factors affect the traffic signal timing at intersections.

These parameters are increasing and decreasing of vehicle speed, the input vehicles volume, type of traffic signal phasing, type of lane, effective green time, cycle length and etc.

Traffic signal timing has optimized due to Iran's standards after identifying the main factors affecting traffic signal timing and considering the simulation.

Software.

Intersection's information related to traffic signal has been obtained from comprehensive traffic company. Finally, a comparison has done between the software and the appropriate software in terms of time, speed and accessibility that could reduce delay and stops and improve capacity and level of service well. 
So traffic signal timing optimization by using simulation and optimization software at the microscopic level transportation planning can make a direct impact to minimize those variables.

Therefore, 2 Intersections of Tehran in 2 different areas (east-west) selected for the case study and studied period in traffic simulation and optimization to address problems related to capacity were in 2014 in this paper.

Traffic data and parameters that related to emissions, and fuel consumption is obtained as ADT in full days of October in studied year. After optimization, the validity and accuracy of project have been used by Aimsun software, and it was observed that traffic signal timing optimization has significant reduction in delays and stops.

\section{Analyzing first simulation with AIMSUN traffic simulator}

Two intersections studied geometrical plan is the first and most important step to enter data into the Aimsun software. In this section, the background of geometric design in AutoCAD format or the images from Google's earth or Google maps put into the Aimsun software as layout before drawing the intersections plan.

After drawing intersections, create centroids, demands, travel center, traffic allocates for every line is like OD matrix and traffic state. In this part geometric design and every phase of line can observe in figure 1 and 2 and also the ratio of capacity to flow in AM and PM peak time, that obtains from first simulation with AIMSUN software are indicated in figure 3 and 4.

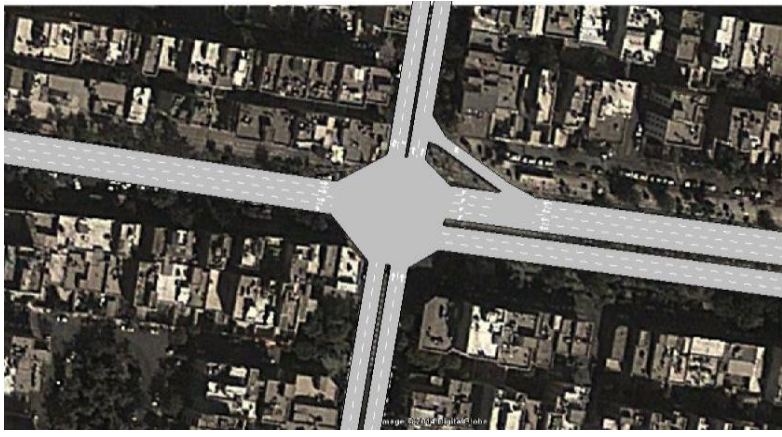

Fig. 1: Geometric Plan of Golabar-Dardasht Intersection.

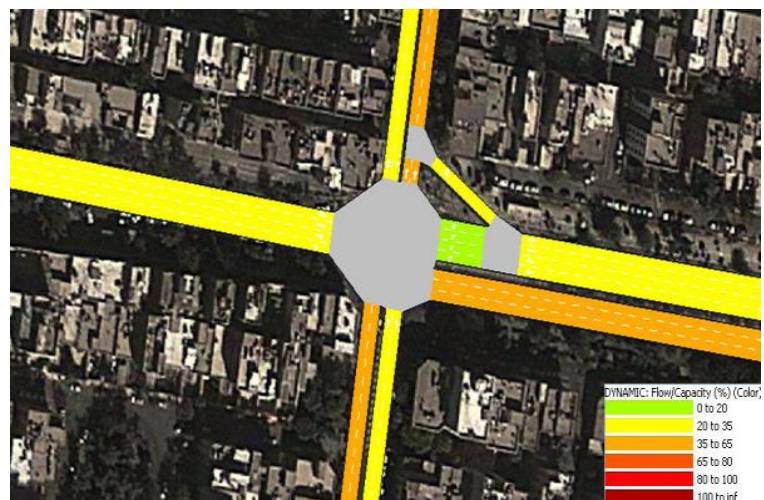

Fig. 3: Percentage of Flow/Capacity, Respectively, During AM and PM Peak Time at Golabar-Dardasht Intersection
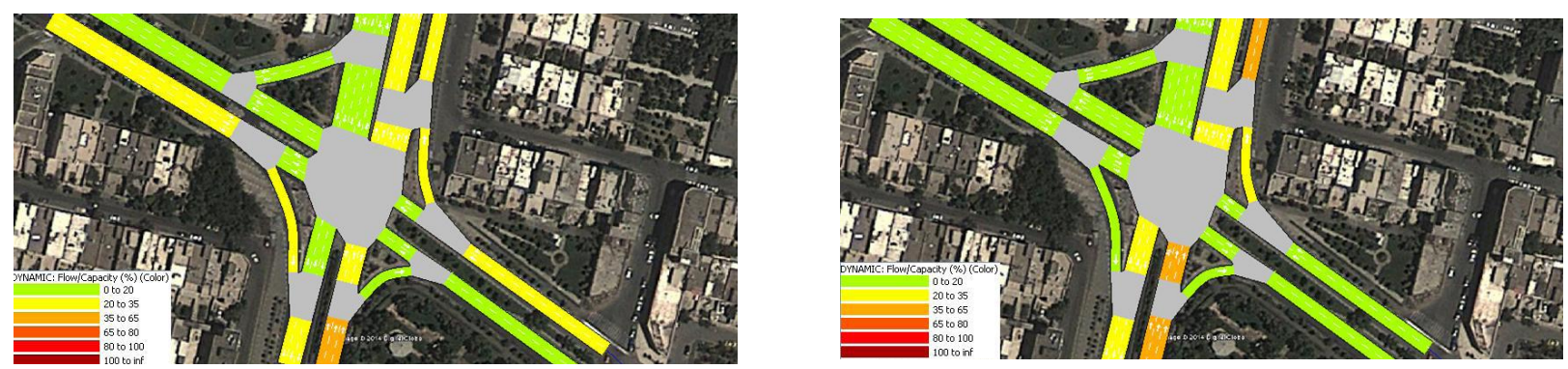

Fig. 4: Percentage of Flow/Capacity, Respectively, During AM and PM Peak Time at Zibadasht-Dehkadeh Intersection

\section{Analyzing and optimization with SYNCHRO software}

After the initial simulation and obtain outputs such as delay time, travelled distance, traffic flow, vehicle average speed, travel time by Aimsun software. Signal timing optimization is done by Synchro software. 
In ultimate step with optimization order in synchro software the possibility of optimizing cycle length is provided automatically.

After intersections optimization the possibility of recording green time, cycle length, level of service in MAP VEIW part for transferring information in second simulation and observing the influence in delay times, level of service, capacity is provided.

Cycle length and two intersection geometry can be observed in figure 5 and 6 during AM and PM peak time after optimization in synchro software. Remember that the level of service respectively change to A and B.
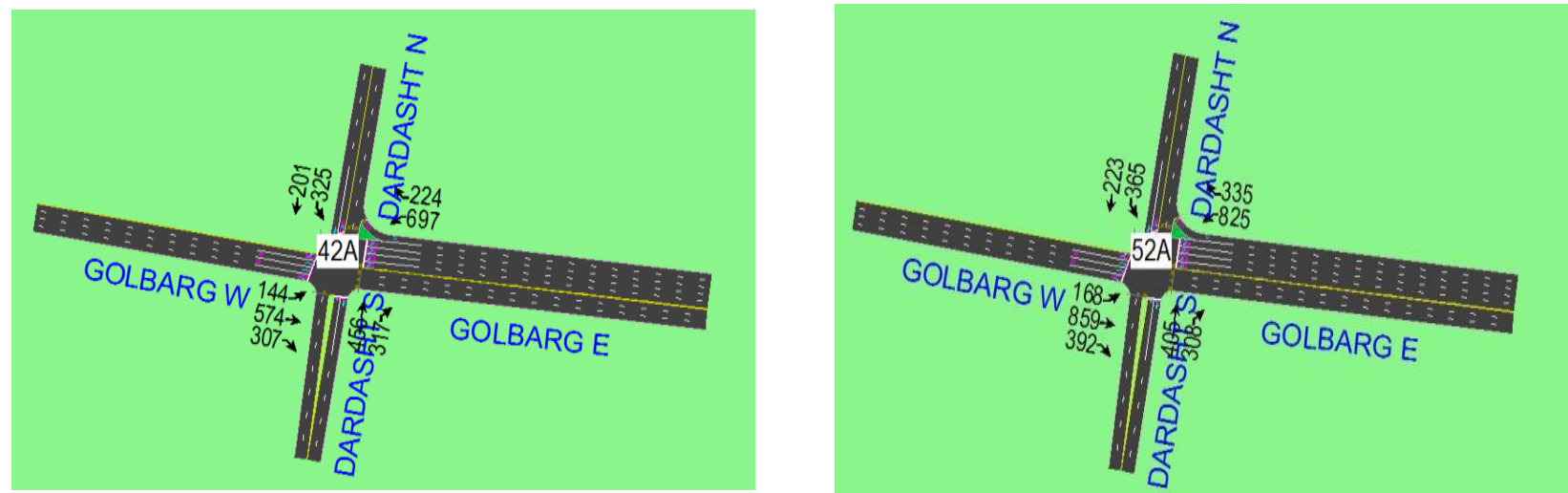

Fig. 5: Cycle Length and Geometric Plan of Golbarg-Dardasht Intersection after Optimization in AM Peak Time by Synchro Software.
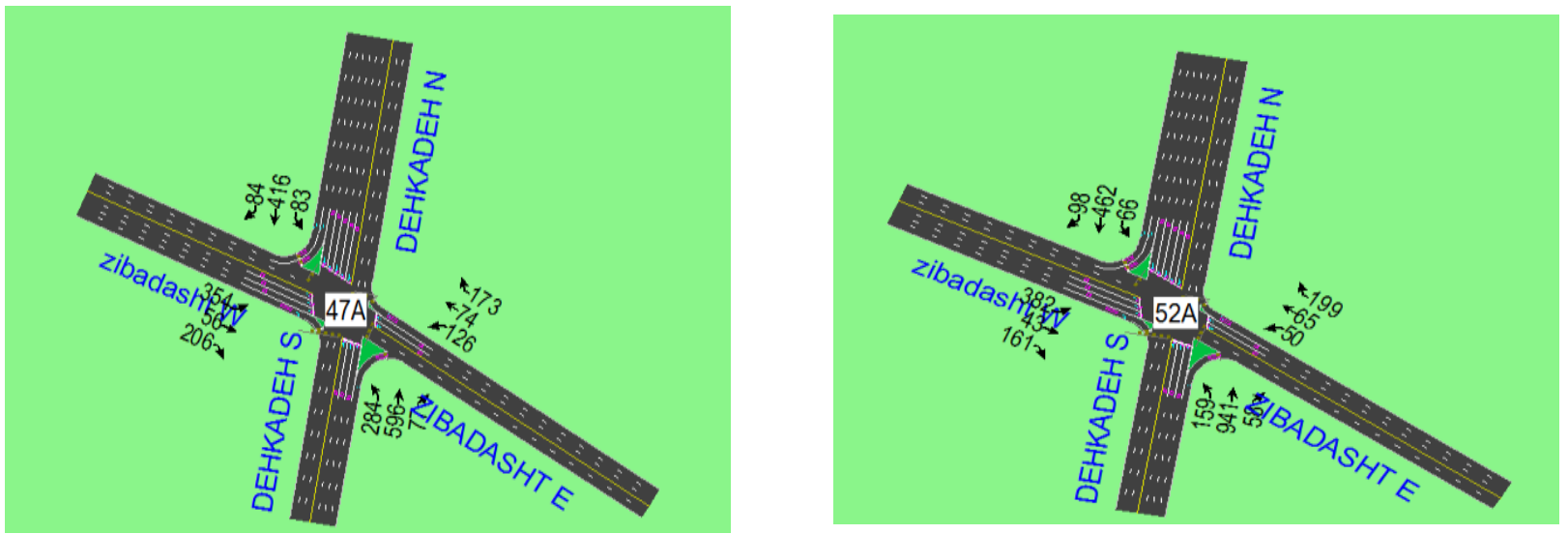

Fig. 6: Cycle Length and Geometric Plan of Zibadasht-Dehkadeh Intersection after Optimization in AM Peak Time by Synchro Software.

\section{Second simulation with AIMSUN after optimization}

The proposed output in table 1 and 2 were obtained after optimizing and putting the traffic information into Aimsun simulator software by second simulation in compare with first simulation.

As observed in tables with decreasing in cycle length, delay and travelled time have been decreased and traffic flow, average speed increase.

It can be seen that the overall amount of delays has been effectively reduced with accuracy in results of Secondary simulation and compare them with traffic flow.

By considering results before and after optimization, changes percent can observe in table 3 and 4 . The positive sign indicates an increase and negative indicate decrease.

By examining changes in tables and considering traffic flow, it can be seen that the amount of delay and travel time have been decreased with improving cycle length and the rate of traffic flow, vehicles average speed increased in both $\mathrm{AM}$ and PM peak hours at each intersection.

Thus providing accurate traffic signal timing optimization algorithms at intersections in Synchro software and observing changes in Aimsun traffic simulator could provide significant reduction in delays and improve a level of service.

After second simulation in Aimsun software the percent of flow /capacity in AM peak times are represented as a sample in figure 7 and 8 . 
Table 1: Output from Second Simulation, Compared with the First Simulation at Golbarg-Dardasht Intersection by Aimsun Software.

\begin{tabular}{|c|c|c|c|c|c|}
\hline Current case & unit & $\begin{array}{l}\text { AM peak time in } \\
\text { first simulation }\end{array}$ & $\begin{array}{l}\text { AM Peak time in } \\
\text { second simulation }\end{array}$ & $\begin{array}{l}\text { PM peak time in } \\
\text { first simulation }\end{array}$ & $\begin{array}{l}\text { PM Peak time in } \\
\text { second simulation }\end{array}$ \\
\hline Delay time & $\mathrm{s} / \mathrm{km}$ & 92.1 & 95.9 & 109.8 & 78.5 \\
\hline Traffic flow & Veh/hr & 2981 & 3565 & 3560 & 2987 \\
\hline $\begin{array}{l}\text { Average } \\
\text { speed }\end{array}$ & $\mathrm{Km} / \mathrm{hr}$ & 28.3 & 28.1 & 26.8 & 29.4 \\
\hline Travel time & $\mathrm{s} / \mathrm{km}$ & 165.5 & 168.4 & 182.6 & 152.1 \\
\hline $\begin{array}{l}\text { Traveled } \\
\text { distance }\end{array}$ & $\mathrm{km}$ & 821 & 993.9 & 994 & 824 \\
\hline
\end{tabular}

Table 2: Output from Second Simulation, Compared with the First Simulation at Zibadasht-Dehkadeh Intersection by Aimsun Software

\begin{tabular}{|c|c|c|c|c|c|}
\hline Current case & unit & $\begin{array}{l}\text { AM peak time in } \\
\text { first simulation }\end{array}$ & $\begin{array}{l}\text { AM Peak time in } \\
\text { second simulation }\end{array}$ & $\begin{array}{l}\text { PM peak time in } \\
\text { first simulation }\end{array}$ & $\begin{array}{l}\text { PM Peak time in } \\
\text { second simulation }\end{array}$ \\
\hline Delay time & $\mathrm{s} / \mathrm{km}$ & 47.8 & 68.1 & 45.4 & 57 \\
\hline Traffic flow & Veh/hr & 2766 & 2757 & 2572 & 2487 \\
\hline $\begin{array}{l}\text { Average } \\
\text { speed }\end{array}$ & $\mathrm{Km} / \mathrm{hr}$ & 36.3 & 33.8 & 36.4 & 35.3 \\
\hline Travel time & $\mathrm{s} / \mathrm{km}$ & 116.6 & 136.9 & 114.8 & 126.6 \\
\hline $\begin{array}{l}\text { Traveled } \\
\text { distance }\end{array}$ & $\mathrm{km}$ & 464.7 & 471.7 & 452.5 & 439 \\
\hline
\end{tabular}

Table 3: Changes Percent of Optimization after Simulation in AIMSUN at Golbarg-Dardasht Intersection

\begin{tabular}{llll}
\hline Current case & unit & Changes percent at AM peak time $\%$ & Changes percent at PM peak time $\%$ \\
\hline Delay time & $\mathrm{s} / \mathrm{km}$ & $-12.65 \%$ & $-14.77 \%$ \\
Traffic flow & $\mathrm{Veh} / \mathrm{hr}$ & $+0.14 \%$ & $+0.2 \%$ \\
Average speed & $\mathrm{Km} / \mathrm{hr}$ & $+4.88 \%$ & $+3.88 \%$ \\
Travel time & $\mathrm{s} / \mathrm{km}$ & $-7.77 \%$ & $-9 \%$ \\
Traveled distance & $\mathrm{km}$ & $-0.01 \%$ & $+0.36 \%$ \\
\hline
\end{tabular}

Table 4: Changes Percent of Optimization after Simulation in AIMSUN at Zibadasht-Dehkadeh Intersection

\begin{tabular}{llll}
\hline & \multicolumn{2}{c}{ Table 4: Changes Percent of Optimization after Simulation in AIMSUN at Zibadasht-Dehkadeh Intersection } \\
\hline Current case & unit & Changes percent at AM peak time\% & Changes percent at PM peak time\% \\
Delay time & $\mathrm{s} / \mathrm{km}$ & $-29.8 \%$ & $-20.35 \%$ \\
Traffic flow & $\mathrm{Veh} / \mathrm{hr}$ & $+0.32 \%$ & $+3.41 \%$ \\
Average speed & $\mathrm{Km} / \mathrm{hr}$ & $+7.39 \%$ & $+3.11 \%$ \\
Travel time & $\mathrm{s} / \mathrm{km}$ & $-14.82 \%$ & $-9.32 \%$ \\
Traveled distance & $\mathrm{km}$ & $-1.48 \%$ & $+3.07 \%$ \\
\hline
\end{tabular}




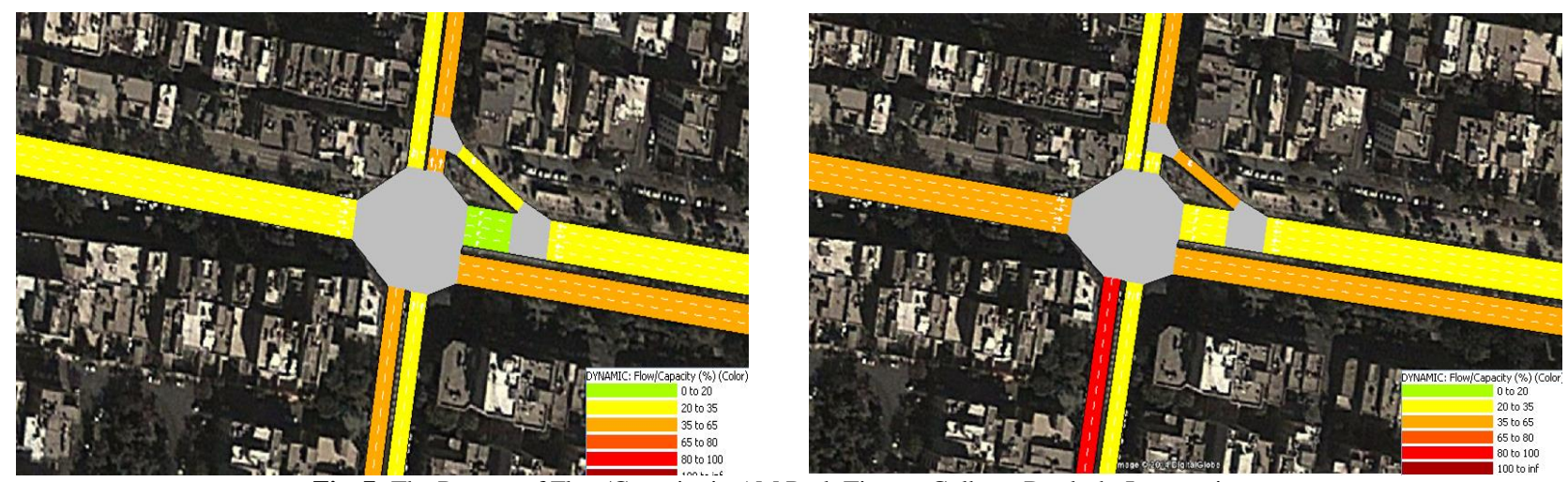

Fig. 7: The Percent of Flow/Capacity in AM Peak Time at Golbarg-Dardasht Intersection.
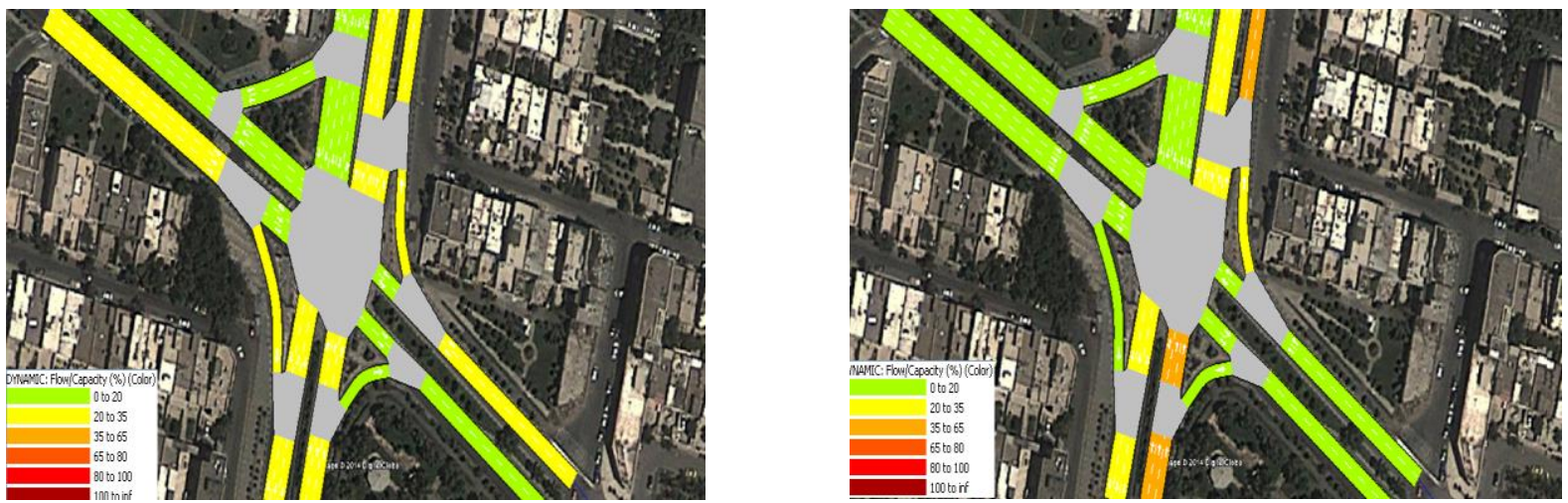

Fig. 8: The Percent of Flow/Capacity in AM Peak Time at Zibadasht-Dehkadeh Intersection.

\section{Conclusion}

Several factors affect the traffic signal timing at intersections. These parameters are increasing and decreasing of vehicle speed, the input vehicles' volume, type of traffic signal phasing, type of lane, effective green time, cycle length and etc.

In this paper, two intersections of East and West of Tehran have been selected, and in two different situations, the flow to capacity ratio of traffic signal timing has been investigated before and after optimization. Simulation by AIMSUN and optimization by SYNCHRO software is done. After optimization can be observed that if before the pre-defined schedule for SCATS, this plan for each intersection of the volume of traffic at peak hours have been optimized, a great reduction in delay and increase in capacity can be observed at intersections.

Optimization has done with synchro and to observe changes, second simulation considered in this study. For sample Changes percent of cycle length, delay times, traffic flow, average speed, travel time, total travel distance respectively $19.23 \%,-14.77 \%,+0.2 \%,+3.88 \%,-9 \%,+0.36 \%$ in AM peak time and $-17.46 \%,-12.65 \%,+0.14 \%,+4.88 \%,-7.77 \%,-0.01 \%$ in PM peak time. Thus the main aspect of the paper which makes it worth worldwide is that we can simulate network based on basic assume before define of traffic signal timing with lower cost, less time and use of expertise activities. Then optimize case study with basic assume to consider cycle length optimization in pre timing of traffic signal.

\section{References}

[1] Hunt P.B., Robertson, D.I., Bretherton, R.D., Winton, R.I., 1981. SCOOT-a traffic responsive method of coordinating signals. Technical Report.

[2] Mauro, V., Taranto, C.D., 1990. Utopia. Control, computers, communications in transportation, 245-252.

[3] Lowrie, P.R., 1982. The Sydney coordinated adaptive traffic system principles, methodology, algorithms. International Conference on Road Traffic Signalling.

[4] Aleksandar Stevanovic, 2013, Optimization of traffic signal timings based on surrogate measures of safety, science direct, Transportation Research Part C (2013).

[5] Byungkyu "Brian" Park, Ilsoo Yun \& Kyoungho Ahn (2009): Stochastic Optimization for Sustainable Traffic Signal Control, International Journal of Sustainable Transportation, 3:4, 263-284.

[6] Pirdavani, A., Brijs, T., Bellemans, T., Wets, G., 2012. A simulation-based traffic safety evaluation of signalized intersections. Presented at 15th International Conference of Road Safety on Four Continents. Transportation Research Board. Abu Dhabi, United Arab Emirates.

[7] Sabra, Z.A., Gettman, D., Henry R.D., Nallamothu, V., 2012. Balancing Safety and Capacity in an Adaptive Signal Control System.

[8] L“ammer, S., Helbing, D., (2010). Self-stabilizing decentralized signal control of realistic, saturated network traffic, Santa Fe Institute.

[9] Lihui Zhang, 2013, Robust signal timing optimization with environmental Concerns,science direct, Transportation Research Part C 29 (2013). 